\title{
Atomic chains of group-IV elements and III-V and II-VI binary compounds studied by a first-principles pseudopotential method
}

\author{
R. T. Senger, S. Tongay, E. Durgun, and S. Ciraci* \\ Department of Physics, Bilkent University, 06800 Ankara, Turkey \\ (Received 11 October 2004; revised manuscript received 27 April 2005; published 12 August 2005)
}

\begin{abstract}
Using the first-principles plane wave pseudopotential method we have studied structural, electronic, and transport properties of atomic chains of group-IV elements and group III-V and group II-VI binary compounds. Several materials which are insulating or semiconducting in bulk are found to be metallic in nanowire structures. Our calculations reveal that monatomic chains of $\mathrm{Si}, \mathrm{Ge}$, and $\mathrm{Sn}$ elements, and of binary compounds such as InP, GaAs, and AlSb, are stable and metallic. On the other hand, compound wires of BN, SiC, GaN, ZnSe, and several others have semiconducting or insulating properties. Ideal mechanical strength calculations show that some of these atomic chains can sustain strains of up to $\varepsilon=0.3$. We have presented $a b$ initio electron transport calculations for $\mathrm{Si}$ and AlP linear chain segments in between $\mathrm{Al}$ electrodes. Conductance of $\mathrm{Si}$ monatomic chains displays some nontrivial features as the number of atoms in the chain is varied or as the chain is strained. In addition to single atomic chain structures, junctions and grid structures of $\mathrm{Si}$ are investigated.
\end{abstract}

DOI: 10.1103/PhysRevB.72.075419 PACS number(s): 73.63. - b, 73.22. $-\mathrm{f}$, 71.20.Nr, 73.40.Jn

\section{INTRODUCTION}

The advent of nanotechnology made it possible to synthesize and manipulate even the ultimately thin wires made of single atomic chains. ${ }^{1-3}$ Metallic nanowires in the form of atomic strings ${ }^{4-11}$ as well as single-shell or multishell helical tubes ${ }^{12-14}$ have been actively studied due to their unusual structural and electronic properties, such as giant Young's modulus ${ }^{15,16}$ and stepwise variation of electronic conductance with the cross section. ${ }^{17-20}$ Aside from fundamental physical interest, nanowires find their relevance in nanotechnology as the potential interconnects between nanodevices. In that respect, conduction and contact properties of stable wire structures are important. Semiconducting and insulating nanowires are also of importance in the context of design and operation of integrated nanoscale devices. Low dimensional forms of materials can have properties quite different from those of their bulk structures. Carbon, for instance, being a good insulator as bulk diamond crystal, can be semiconducting or metallic in the form of nanotubes. The linear atomic chain of carbon is even a better conductor than gold chain. ${ }^{21,22}$ Although nanowires of metallic elements, ${ }^{23}$ carbon nanotubes, ${ }^{24}$ and atomic strings of carbon ${ }^{22,25-28}$ have been investigated extensively, studying similar forms of other group-IV elements ( $\mathrm{Si}, \mathrm{Ge}, \mathrm{Sn}$ ) and III-V (such as BN, $\mathrm{AlP}, \mathrm{GaAs}$, etc.) and II-VI (CdS, $\mathrm{ZnSe}$, etc.) compounds are relatively recent. ${ }^{29-39}$ These materials, which have covalent or polar semiconductor crystal forms in bulk, as well as quasi-two-dimensional and finite cross-section onedimensional (1D) geometries, ${ }^{40,41}$ are used in important optoelectronic applications. Their string forms are expected to exhibit some useful properties to be exploited in future electronic devices.

In this paper we present a systematic study of string structures of widely used III-V and II-VI compound materials and some of group-IV elements in order to reveal their fundamental structural and electronic properties. ${ }^{44}$ The synthesis of stable finite-size carbon ${ }^{25,26}$ and silicon ${ }^{39}$ atomic chains has been one of the principle factors that motivated the present theoretical study. The atomic chain structures that we consider constitute the ultimate lower limit in thickness of the silicon and compound nanowires that have been studied actively ${ }^{40-43}$ There are several forms of atomic ordering in 1D geometry, which are found by theoretical calculations to yield stable structures, which we label "atomic strings." We have considered various string structures, such as linear chain (LC), planar zigzag with wide (WZ) and narrow (NZ) apex angles, and cross-junction, planar, and buckled-grid structures. Figure 1 presents a schematic description of various string structures that are considered in this paper. The bonding, stability, as well as mechanical, electronic, and transport properties of these structures are analyzed using first-principles methods. In particular, we found that planar grids made of silicon strings are stable and could have useful nanoscale device-integration applications if they can be synthesized. The bonding characters in string structures are found to be quite different from their counterparts in the bulk crystals and underly their unusual physical properties.

Recently, the ultimately thin atomic chains of $\mathrm{Si}$ have been the subject matter of several theoretical calculations. Using a model of jellium electrodes, and by solving the scattering matrix of the system, Mozos et al. ${ }^{4}$ have computed the conductance of $\mathrm{Si}$ atomic chains with up to eight atoms. In a similar account, Okano et al. ${ }^{10}$ performed an eigenchannel analysis of the conductance of $\mathrm{Si}$ and $\mathrm{Al}$ atomic wires again using jellium electrodes. We have investigated conductance variations of monatomic $\mathrm{Si}$ chains in between $\mathrm{Al}$ electrodes as functions of chain length, contact geometry, and applied strain, and found some nontrivial variations. Additional atoms bound to silicon chains modify their structural and conductance properties.

\section{METHOD}

We have performed self-consistent-field total energy and electronic structure calculations using the first-principles 
Linear Chain (LC) Wide Zigzag (WZ) Narrow Zigzag (NZ)
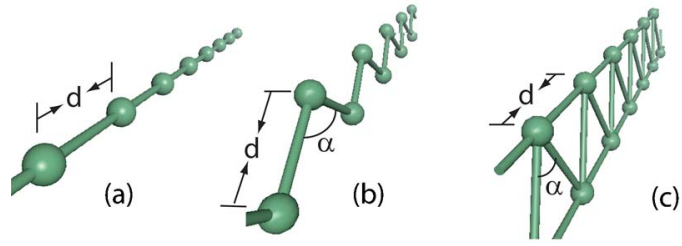

Si adatom on Si chain

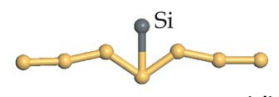

(d)

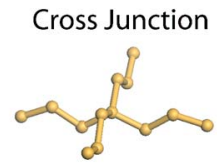

(e)
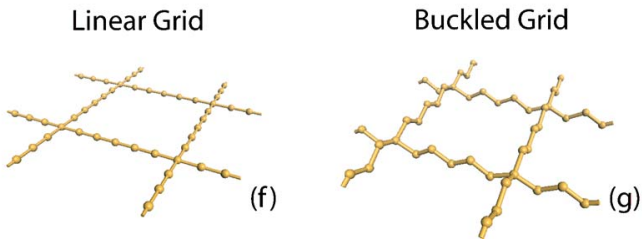

FIG. 1. (Color online) Schematic description of various string structures: linear and zigzag chains, junctions, and grids. In the case of compounds, anion and cation atoms alternate in the strings. $d$ is the bond length; $\alpha$ is the apex angle.

pseudopotential plane wave method ${ }^{45,46}$ within density functional theory ${ }^{47}$ (DFT). Generalized gradient approximation ${ }^{48}$ (GGA) and local density approximation ${ }^{49}$ (LDA) have been used. It is known that LDA leads to overbinding. The LDAcalculated lattice parameters of the chains are about $0.1 \AA$ smaller than those calculated using GGA, and the band gaps are about $0.1-0.3 \mathrm{eV}$ larger. While DFT-based methods are known to provide reliable predictions for the ground state properties of structures made of group-IV elements and III-V and II-VI compounds, the excited states are not well treated. In particular the band gaps are underestimated. The eigenvalues of Kohn-Sham equations ${ }^{47}$ are only approximations to the actual band energies. The limitations of DFT in predicting excited states and excitonic effects can be improved by employing many-body corrections within $G W{ }^{50}$ In this work we report GGA-calculated values unless denoted otherwise.

The structures we consider are treated within supercell geometries. The Brillouin zone of the 1D structures has been sampled by using the Monkhorst-Pack k-point sampling scheme, ${ }^{51}$ with a sufficient number of $\mathbf{k}$ points as determined by convergence tests. For the ultrasoft pseudopotentials ${ }^{46,52}$ used in our calculations, the wave functions are represented by plane waves with cutoff kinetic energies of $300 \mathrm{eV}$ $<\hbar^{2}|\mathbf{k}+\mathbf{G}|^{2} / 2 m<400 \mathrm{eV}$. All the atomic positions and lattice parameters of the structures have been optimized by minimizing the total energy, forces on atoms, as well as the stress on the structure. We performed an analysis of stability by reoptimizing the structures after displacing individual atoms from their original optimized configuration. Furthermore, $a b$ initio molecular dynamics (MD) calculations have been carried out for all structures at high temperatures $(800 \mathrm{~K} \leqslant T \leqslant 1200 \mathrm{~K})$ for a large number of femtosecond time steps.

As for the analysis of quantum ballistic conductance, we have used a recently developed $a b$ initio transport software
(TRANSIESTA-C) based on DFT, localized basis sets, and a nonequilibrium Green's function (NEGF) formalism. ${ }^{53}$ The equilibrium conductance values of the structures are calculated in the usual electrode-device-electrode geometry. The conductance of the device has been calculated as

$$
G(E)=\frac{2 e^{2}}{h} \operatorname{Tr}\left(\Gamma_{l} G^{r} \Gamma_{r} G^{a}\right)
$$

after iteratively solving nonequilibrium Green's function and DFT equations. ${ }^{53}$ In the above equation, $G^{r}$ and $G^{a}$ are the retarded and advanced Green's functions, $\Gamma_{l}$ and $\Gamma_{r}$ are the coupling functions to the left and right electrodes, respectively. DFT is employed to describe exchange correlation and charging effects, as well as the atomic structure of both the device and the electrodes in a self-consistent manner. Unlike conventional DFT simulation schemes, in order to account for the infinite and open nature of the system due to the electrodes, DFT is used in combination with NEGF formalism. The latter provides the means for representing the effects of infinite electrodes in the form of self-energy terms.

For the sake of consistency, in our transport calculations the structure geometries optimized by plane wave method are supplied to TRANSIESTA-C, which uses localized numerical basis sets. We have employed double- $\zeta$ parameter numerical basis sets which are usually comparable to wellconverged plane wave basis sets. To check the compatibility of the two methods (plane wave versus localized basis set) we have performed several test calculations which led to satisfactory results. For example, the equilibrium lattice constant of the Si linear chain (Si-LC) is obtained as $2.172 \AA$ using plane wave and $2.189 \AA$ A using local basis, the discrepancy being less than $1 \%$. Correspondingly, the induced forces on the atoms due to the minor differences between the structure optimization results of the two methods are also well below acceptable limits.

We have studied Si and AlP linear chains as representative sample systems of finite-length chains. In both cases, Al bars with a finite cross section oriented in the [001] direction have been taken as electrodes. The semi-infinite electrodes have a unit cell of nine $\mathrm{Al}$ atoms in two layers of four and five atoms. We consider two types of contact geometries, where the terminal layers of electrodes have either four or five Al atoms. In the former case, the ends of the Si or AlP chains are positioned at the "hollow site," but in the latter case, at the "top site" of the electrodes (see Fig. 2). Depending on the symmetry of the system, three or four atomic layers of the left and right electrodes are included in the device region in order to provide tractable boundary conditions at the interface of the device and electrode regions. The conductance of atomic-chain devices has been calculated for unrelaxed, relaxed, and strained geometries. Further details on the conductance calculations are given in Sec. IV.

\section{ATOMIC AND ELECTRONIC STRUCTURE}

\section{A. Atomic structure and mechanical properties}

We first consider three types of periodic string structures, namely, infinite linear chain, LC, and planar zigzag struc- 

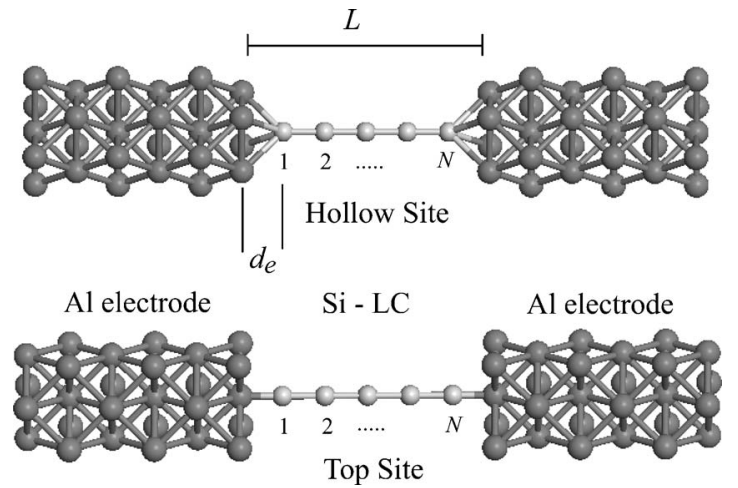

FIG. 2. $N$-atom Si-LC in between Al (100) electrodes for two different binding geometries: hollow site and top site. The distance of the chain terminal atoms to the surface plane of the electrodes is denoted by $d_{e}$.

tures, WZ and NZ. The binding energy per basis for a given lattice parameter $c$ is calculated from the expression $E_{I}(c)$ $=\left\{E_{T}[A C, c]-E_{T}[A]+E_{T}[C]\right\}$ in terms of the total energy of the free anion atom $A$, free cation atom $C$, and the total energy of the optimized chain structure of the basis $A C$. In the case of group-IV elements, $A=C$. The minimum of $E_{I}(c)$ (the negative of it is specified as binding energy $E_{b}$ ) corresponds to an optimized atomic structure for a given geometry.

In Fig. 3 we present the variation of $E_{I}(c)$ for the string structures of $\mathrm{C}, \mathrm{Si}, \mathrm{AlP}, \mathrm{GaAs}, \mathrm{SiC}$, and CdTe as a function of the lattice parameter $c$ optimized for LC and planar zigzag

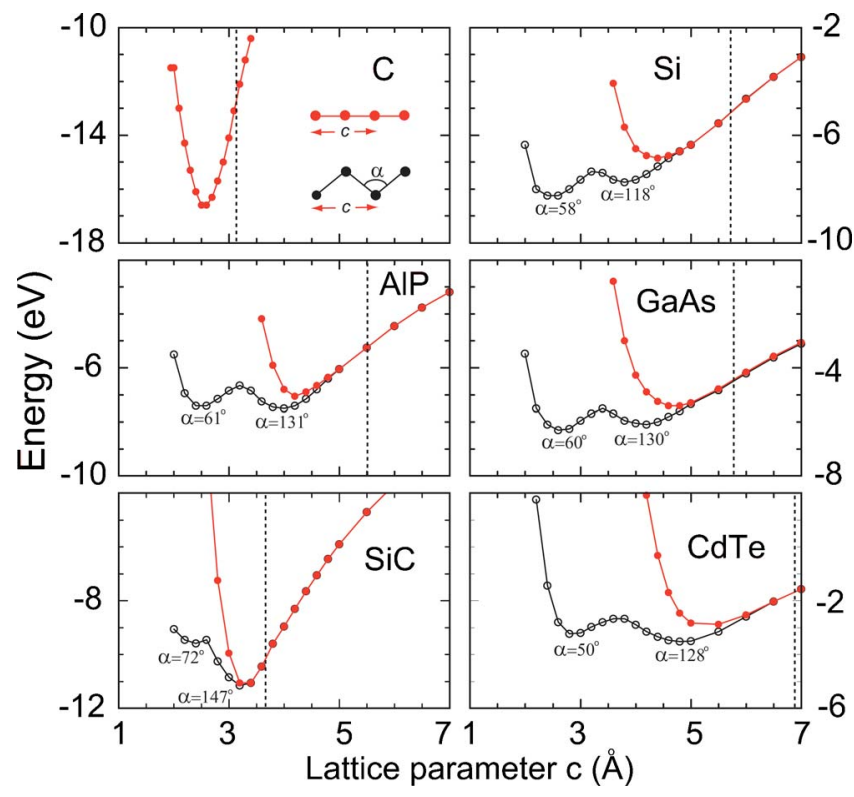

FIG. 3. (Color online) Calculated energy $E_{I}(c)$ per two-atom basis of some elemental and compound atomic chains as functions of lattice parameter. Solid circles correspond to linear geometries and open circles to planar zigzag geometries. The curves are guides for the eye. The optimized apex angle values at the local minima are given. The vertical dashed lines denote the boundary of the stability domain of the strained chains with respect to longitudinal deformations. geometries. Linear chain structure is a precursor to other planar zigzag strings. Except for $\mathrm{C}$ and BN, the LC structure of group-IV elements and the compounds appear to be metastable, since the WZ structure occurs at a slightly lower energy. As a freestanding chain, small transversal displacements of alternating atoms may transform LC to a WZ structure. ${ }^{54}$ On the contrary, WZ structures appear to be a stable structure for chains of group-IV elements and III-V and II-VI compounds, except for $\mathrm{C}$ and $\mathrm{BN}$ chains.

The mechanical strength of the atomic chains can be evaluated using their binding energy variations as functions of the lattice parameter. Strained chains become linear as seen by the merging of the energy curves for the zigzag and linear geometries at large values of $c$. The maximum strain that can be applied to an atomic chain before breaking is obtained by considering the point where longitudinal acoustic mode has zero sound velocity. That is equivalent to finding the lattice constant at which $d^{2} E_{I} / d c^{2}=0$. The vertical dashed lines in Fig. 3 denote those boundaries where instabilities due to long-range longitudinal deformations show up. ${ }^{8}$ The analysis shows that the maximum strain values that can be sustained by the atomic chains are $0.23,0.28,0.31$, 0.23, 0.12, and 0.31 for $\mathrm{C}, \mathrm{Si}, \mathrm{AlP}, \mathrm{GaAs}, \mathrm{SiC}$, and CdTe, respectively. We should note, however, that instabilities due to short wavelength perturbations may limit these stability domains against axial strains, a formal analysis of which requires calculation of the phonon modes of the chain structures. $^{8}$

In Table I we summarize the structural properties of the three string structures LC, WZ, and NZ and compare their binding energies $E_{b}$ with those of their parent bulk crystals. Although the coordination number of atoms is reduced significantly in one-dimensional chains as compared to their bulk crystals, the binding energies per atom (per basis for compounds) are still high as seen in Table I. The character of the strong binding is given by the formation of double bonds between adjacent atoms; one of them, the $\sigma$ bond, is composed of $s$ - and $p_{z}$-valence orbitals. The second bond is the $\pi$ bond made by $p_{x}$ and $p_{y}$ atomic orbitals perpendicular to the chain axis. The character of bonding is covalent in chains made of group-IV elements, but gains some polarity in compound wires due to transfer of charge from cation atoms of group III (II) to anion atoms of group V (VI). Charge density contour plots of the bonds in selected chains depict the character of the bond as shown in Fig. 4. Similar to their bulk crystals, the covalent character of the bonds in $\mathrm{Si}$ and $\mathrm{Ge}$ linear chains is reflected by the accumulation of bond charge between two nearest-neighbor atoms and by the symmetry of the contour lines. In compound chains, however, the symmetry of charge density is altered in favor of anions. As seen in the figure, SiGe compound chain has highly covalent bonding character since the asymmetry in the bonding charge density is quite minor.

Another consequence of the double-bonding pattern is reflected in mechanical properties of these ultimate onedimensional structures. We note that LC may be unstable against transverse displacement of atoms, but is stiff against longitudinal strain. In actual cases finite atomic chains should be considered in between electrodes which provide an external strain stabilizing the chains in LC geometry. The 
TABLE I. Summary of calculated binding energies and structural parameters of LC, WZ, and NZ atomic chains. $E_{b}$ is the binding energy in eV/atom (eV/basis for compounds). $\alpha$ is the apex angle in degrees. $d$ is the bond length in $\AA$. The last column is the bulk cohesive energies of the elemental or compound semiconductors in $\mathrm{eV}$.

\begin{tabular}{|c|c|c|c|c|c|c|c|c|c|c|}
\hline & \multicolumn{3}{|c|}{$\mathrm{LC}$} & \multicolumn{3}{|c|}{ WZ } & \multicolumn{3}{|c|}{$\mathrm{NZ}$} & \multirow{2}{*}{$\begin{array}{r}\text { Bulk } \\
E_{b}\end{array}$} \\
\hline & $E_{b}$ & $\alpha$ & $d$ & $E_{b}$ & $\alpha$ & $d$ & $E_{b}$ & $\alpha$ & $d$ & \\
\hline $\mathrm{Si}$ & 3.4 & 180 & 2.2 & 3.9 & 118 & 2.2 & 4.1 & 58 & 2.5 & 5.4 \\
\hline $\mathrm{Ge}$ & 3.0 & 180 & 2.4 & 3.4 & 116 & 2.4 & 3.6 & 61 & 2.6 & 4.5 \\
\hline $\mathrm{Sn}$ & 2.5 & 180 & 2.7 & 2.8 & 114 & 2.7 & 3.2 & 60 & 3.0 & 3.8 \\
\hline $\mathrm{SiC}$ & 11.1 & 180 & 1.7 & 11.2 & 147 & 1.7 & 9.5 & 72 & 1.9 & 15.2 \\
\hline $\mathrm{BN}$ & 16.2 & 180 & 1.3 & & & & & & & 17.5 \\
\hline $\mathrm{GeSi}$ & 6.3 & 180 & 2.2 & 7.5 & 118 & 2.2 & 8.2 & 59 & 2.5 & 10.0 \\
\hline AlP & 7.0 & 180 & 2.1 & 7.5 & 131 & 2.2 & 7.4 & 61 & 2.4 & 10.4 \\
\hline GaAs & 5.5 & 180 & 2.3 & 6.1 & 130 & 2.3 & 6.3 & 60 & 2.6 & 8.3 \\
\hline $\mathrm{InSb}$ & 4.5 & 180 & 2.8 & 5.0 & 123 & 2.7 & 5.3 & 57 & 3.1 & 6.9 \\
\hline $\mathrm{ZnSe}$ & 4.0 & 180 & 2.2 & 4.6 & 133 & 2.3 & 3.9 & 56 & 2.5 & 6.1 \\
\hline $\mathrm{CdTe}$ & 2.9 & 180 & 2.7 & 3.5 & 128 & 2.7 & 3.2 & 50 & 3.3 & 4.9 \\
\hline
\end{tabular}

tensile force on the chain structure is calculated using $F_{z}$ $=-\partial E_{b}(c) / \partial c$. In order to avoid ambiguity in definitions, we choose to analyze the elastic stiffness of the chains using the quantity $\kappa=d^{2} E /\left.d \epsilon^{2}\right|_{\epsilon=0}$ (the second derivative of the strain energy per atom with respect to the axial strain at equilibrium) rather than the Young modulus, which requires assign-
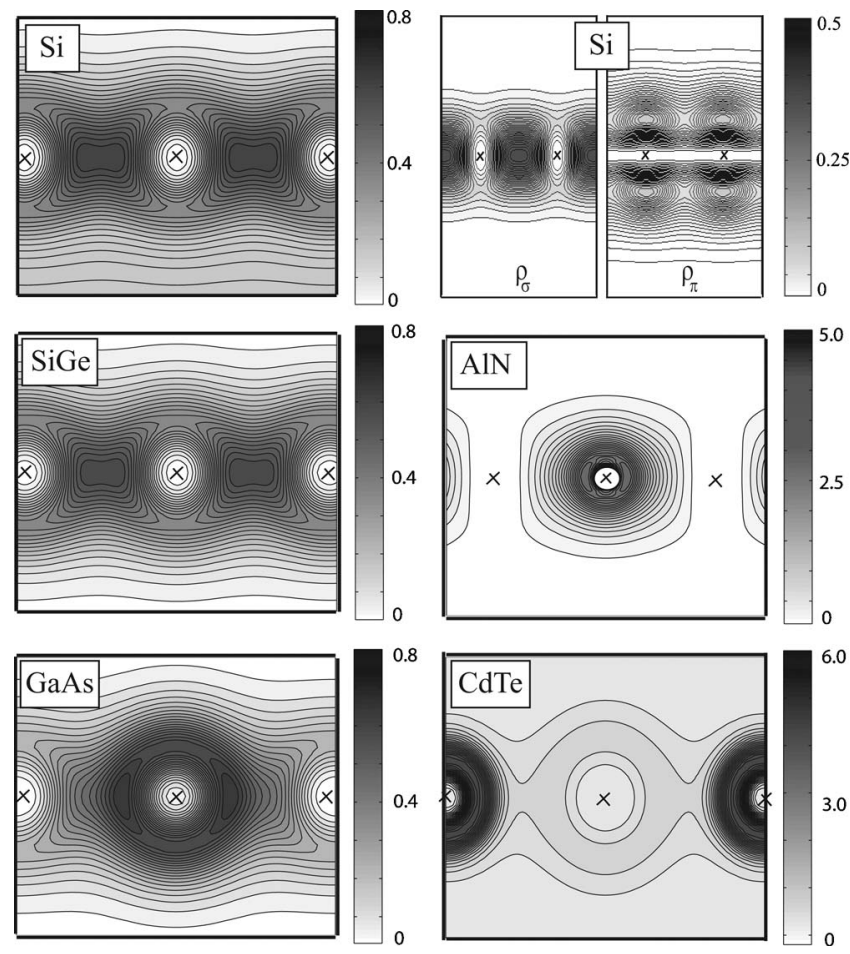

FIG. 4. Contour plots of total charge density of $\mathrm{Si}, \mathrm{SiGe}, \mathrm{AlN}$, GaAs, and CdTe linear chains in the planes passing through the atoms. For Si-LC only, the charge densities $\rho_{\sigma}$ and $\rho_{\pi}$ associated with $\sigma$ bands and $\pi$ bands are also shown. The atomic positions are denoted by crosses. The contour values can be deduced from the gray scales. ing an effective thickness to the wires. The numerical value of $\kappa$ is calculated to be $32 \mathrm{eV}$ for Si-LC. An AlP linear chain (AlP-LC) is found to be stiffer than Si with $\kappa=100 \mathrm{eV}$. As for comparison, a carbon linear chain (C-LC), has $\kappa$ $=119 \mathrm{eV}, 22$ and several single-wall carbon nanotubes are calculated to have $\kappa$ values in the range $52-60 \mathrm{eV} .^{55}$

Having discussed LC, WZ, and NZ structures, we now consider two-dimensional string structures of $\mathrm{Si}$. Whether $\mathrm{Si}$ atomic chains can form $T$ junctions and cross junctions is a relevant question to address, because the realization of such junctions is a prerequisite for the generation of $\mathrm{Si}$ atomic networks and grids, which may have potential applications in molecular electronics. Ab initio DFT calculations predict that a variety of such junctions are possible.

For the branching of Si chains to form junctions, the attachment of an additional atom should be energetically favorable. Figure 1(d) shows the geometry of a Si atom attached to an initially linear, seven-atom $\mathrm{Si}$ chain. In that symmetric configuration, the external $\mathrm{Si}$ atom is bound to the center atom of the chain at the top site, causing a deformation in the chain. A $T$ junction can form if a perpendicular chain develops from that adatom. When the external $\mathrm{Si}$ atom is located in a bridge site of a short $\mathrm{Si}$ chain, the final configuration of the structure looses its one-dimensional character through clustering. In the case of cross-junction formation of two Si chain segments, the most favorable configuration is found to be the junction of two buckled chains by sharing a single $\mathrm{Si}$ atom at the center, as shown in Fig. 1(e). In that particular $7 \times 7$ atomic junction the average binding energy of the atoms is calculated to be $3.7 \mathrm{eV} /$ atom. The center atom makes bonds with its four nearest neighbors in perfect tetragonal angles, and the apex angles in the chains are $\sim 120^{\circ}$, corresponding to the Si-WZ form.

A periodic combination of such junctions can produce grid and network structures made of $\mathrm{Si}$ atomic strings. For example, a $6 \times 6$ linear atomic grid of silicon, as shown in Fig. 1(f), has a binding energy of $\sim 3.7 \mathrm{eV} /$ atom. However, it is found that the linear grid structure can lower its energy 


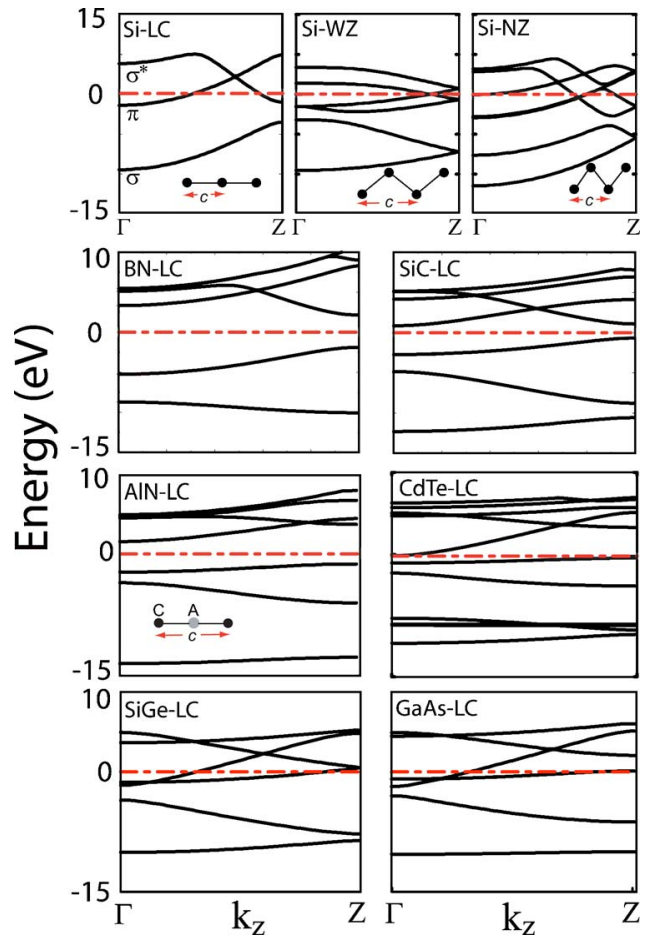

FIG. 5. (Color online) Calculated energy band structures of linear WZ and NZ chains of $\mathrm{Si}$ and $\mathrm{BN}, \mathrm{SiC}, \mathrm{AlN}, \mathrm{CdTe}, \mathrm{SiGe}$ and GaAs linear chains. Si-LC bands are drawn for single-atom unit cell, all other structures have two-atom unit cells. Fermi levels are denoted by dashed lines.

through transforming its linear branches into zigzag chains. The buckled grid structure, shown in Fig. 1(g), has a higher binding energy (4.0 eV/atom).

\section{B. Electronic band structure}

The electronic band structures of three $\mathrm{Si}$ chain geometries and of some selected compound linear chains are presented in Fig. 5. Around the Fermi level, the generic band structure of these atomic wires with two-atom unit cells is composed of two $\sigma$ bonding bands, two doubly degenerate nonbonding $\pi$ bands, and antibonding $\sigma^{*}$ bands. The Si-LC has a doubly degenerate $\pi$ band and a $\sigma^{*}$ band crossing the Fermi level leading to $3 G_{0}$ a conductance $\left(G_{0}=2 e^{2} / h\right.$ is the quantum of conductance). Interestingly, the conductance first drops to $2 G_{0}$ and then increases to $4 G_{0}$ as the Si chain buckles to WZ and NZ structures, respectively. In the set of LCs presented in Fig. 5, except for $\mathrm{BN}, \mathrm{SiC}$, and $\mathrm{AlN}$ the periodic chains are metallic. The insulating AlN linear chain (AlNLC) has an indirect band gap of $2.8 \mathrm{eV}$. The BN linear chain (BN-LC) has a wider gap of $4.0 \mathrm{eV}$. In $\mathrm{Si}, \mathrm{Ge}, \mathrm{SiGe}$, and GaAs chains the Fermi levels cross both a degenerate $\pi$ band and a $\sigma^{*}$ band, giving rise to three channels for the ballistic conductance. In these LCs dipping of $\sigma^{*}$ band below the Fermi level partially delocalizes the double bond, lowering the cohesive energy accordingly. Another consequence of crossing of the Fermi level by three bands is the prohibition of Peierls distortion in these chains through dimerization, unlike the case of carbon LC where only the half-filled $\pi$

\begin{tabular}{|c|c|c|c|}
\hline $\begin{array}{c}\mathrm{Si} \\
\mathrm{M}: 3\end{array}$ & $\begin{array}{c}\mathrm{InP} \\
\mathrm{M}: 3\end{array}$ & $\begin{array}{c}\text { InAs } \\
\mathrm{M}: 3\end{array}$ & $\begin{array}{c}\mathrm{CdTe} \\
\mathrm{M}: 3\end{array}$ \\
\hline $\begin{array}{c}\mathrm{Ge} \\
\mathrm{M}: 3\end{array}$ & $\begin{array}{c}\mathrm{InSb} \\
\mathrm{M}: 3\end{array}$ & $\begin{array}{c}\mathrm{AlSb} \\
\mathrm{M}: 3\end{array}$ & $\begin{array}{c}\mathrm{CdSe} \\
\mathrm{I}=0.4\end{array}$ \\
\hline $\begin{array}{c}\mathrm{Sn} \\
\mathrm{M}: 3\end{array}$ & $\begin{array}{c}\mathrm{GaAs} \\
\mathrm{M}: 3\end{array}$ & $\begin{array}{c}\mathrm{AlAs} \\
\mathrm{I}=0.1\end{array}$ & $\begin{array}{c}\mathrm{ZnTe} \\
\mathrm{I}=0.1\end{array}$ \\
\hline $\begin{array}{c}\mathrm{GeSi} \\
\mathrm{M}: 3\end{array}$ & $\mathrm{GaP}$ & $\mathrm{AlP}$ & $\mathrm{ZnSe}$ \\
$\mathrm{M}: 3$ & $\mathrm{I}=1.1$ & $\mathrm{I}=0.8$ \\
\hline $\mathrm{SiC}$ & $\mathrm{GaN}$ & $\mathrm{AlN}$ & $\mathrm{ZnS}$ \\
$\mathrm{I}=2.4$ & $\mathrm{I}=1.8$ & $\mathrm{I}=2.7$ & $\mathrm{I}=1.6$ \\
\hline
\end{tabular}

FIG. 6. A systematic presentation of the electronic character of linear chain structures. The cells associated with metallic $(M)$ chains are painted in gray and the ballistic conductance values are given in units of conductance quantum $2 e^{2} / h$. The numerals for insulating $(I)$ chains are the values of the energy band gap in $\mathrm{eV}$.

bands are crossed by the Fermi level at $k_{z}=\pi / 2 c$. Therefore, the carbon LC structure is susceptible to Peierls instability, the degree and observability of which, however, is still controversial. $^{22,28}$ Of the II-VI compound linear chains we considered, only CdTe is metallic with $3 G_{0}$. Other II-VI compound linear chains are insulating with entirely filled $\pi$ bands. It should be noted that the information provided by the band structure of the LC structures about their conductance properties is limited to their infinitely long forms. The conductance of actual, finite-sized chains depends on the length, type, and shape of electrodes and the quality of contacts.

The charge densities associated with the $\sigma$ bands and $\pi$ bands of Si-LC are shown in Fig. 4. The symmetry of the charge distributions represents the orbital composition of these bands. In the AlP chain, for instance, the valence bands are dominantly derived from atomic $p$ orbitals.

The electronic character of the linear chain structures is summarized in Fig. 6. The materials are grouped into columns; the group-IV elements and their compounds are in column 1, III-V compounds are in columns 2 and 3, and II-VI compounds form the last column. All II-VI compounds are semiconducting or insulating except for the CdTe linear chain which is metallic. The ballistic conductance values of metallic chains are $3 G_{0}$ as discussed above. In general, we observe that the metallic character of the chains increases with the increasing row number of elements in the periodic table. Nevertheless, in view of the fact that LDA and GGA underestimate the band gap, one has to be cautious in attributing metallicity to CdTe in Fig. 6 because $E_{F}$ is marginally crossing the energy bands. The vanishing band gap may be due to GGA underestimating its value. The predictions of GGA that SiGe LC and GaAs LC are metallic appear to be valid though.

\section{BALLISTIC ELECTRON TRANSPORT THROUGH FINITE-SIZE LINEAR CHAINS}

Having presented the structural and electronic properties of infinite elemental and compound chains, we now focus on 

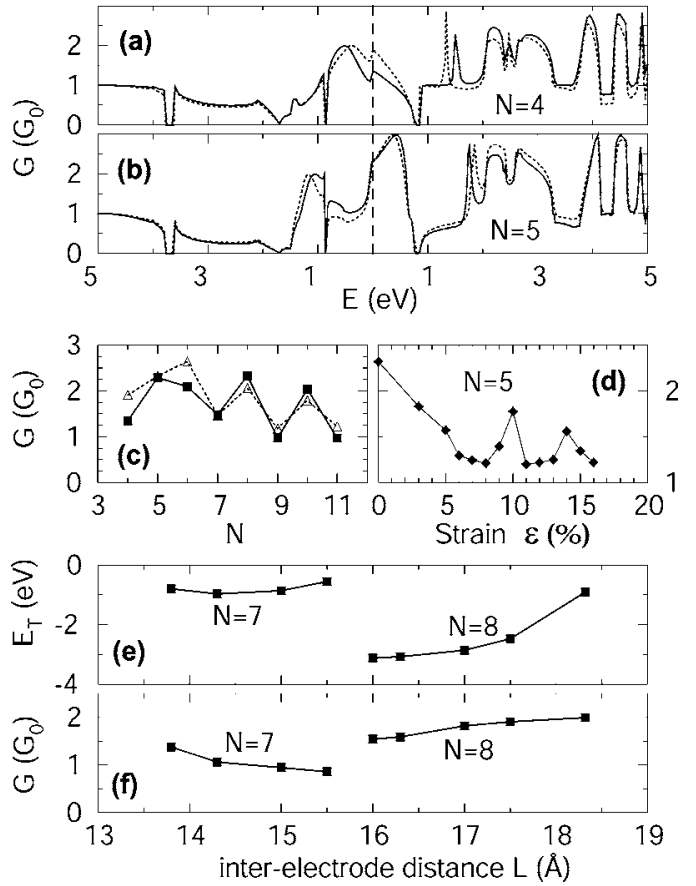

FIG. 7. Calculated conductance plots of $\mathrm{Si}$ atomic chains as connected to $\mathrm{Al}$ (100) electrodes in the hollow-site geometry. (a) and (b) The conductance spectrum of four- and five-atom Si chains, respectively. The dashed curves are the results for unrelaxed chains, assuming lattice parameters of the infinite chains. (c) The variation of equilibrium conductance, $G\left(E=F_{F}\right)$, with the number of Si atoms in the chain, $N$. Again, the dashed plot is for unrelaxed chains. (d) The variation of equilibrium conductance in a five-atom Si-LC with uniform elongation of the structure. (e) The variation of the total energy in seven- and eight-atom Si zigzag chains as functions of the interelectrode distance. For each data point the atomic positions are reoptimized. The reference energy is chosen arbitrarily for each curve. (f) Corresponding conductance variations in the seven- and eight-atom $\mathrm{Si}$ zigzag chains as functions of the interelectrode distance.

conductance calculations of finite-length monatomic linear chains. We consider finite Si-LC and AlP-LC as prototypes of elemental and compound chain structures, respectively. Most of the calculations are made for the hollow-site binding configuration. The electrodes are taken as finite, crosssection, semi-infinite Al bars as schematically shown in Fig. 2. The electrodes are assumed to be rigid having their bulk lattice parameters. For comparison purposes we have performed conductance calculations for some nonoptimized as well as reoptimized structures. In the former case we take the equilibrium interatomic distance in the linear chains as their values in the infinite chains, that is $d_{\mathrm{Si}-\mathrm{Si}}=2.17 \AA$, and $d_{\mathrm{Al}-\mathrm{P}}=2.08 \AA$. Similarly, in those cases we fixed the equilibrium distance of end atoms in the chain to the surface of the electrodes $\left(d_{e}\right.$ in Fig. 2$)$ as $d_{\text {Si-elec. }}=1.56 \AA$ [the equilibrium distance of a $\mathrm{Si}$ adatom on an $\mathrm{Al}(100)$ surface $\left.^{56}\right]$ and $d_{\text {AlP-elec. }}=2.0 \AA$. It is found that relaxation of the chains slightly modifies the conductance patterns, but their qualitative forms are not much affected.

In Fig. 7 we display the results of our calculations on the conductance of finite-size $\mathrm{Si}$ chains in between $\mathrm{Al}$ electrodes.
In panels (a) and (b), the conductance of chains made of four and five $\mathrm{Si}$ atoms is presented. The conductance plots of nonoptimized structures in the electrode-chain-electrode geometries are also shown as dashed curves for comparison. The relaxation of finite-length chains in the linear chain geometry leads to modified bond lengths (e.g., the five-atom Si chain has 2.25-2.14-2.14-2.25 $\AA \mathrm{Si}-\mathrm{Si}$ and shorter $\mathrm{Si}$ - Al distances instead of uniform bond lengths); however, the resulting conductance patterns are the same qualitatively and only slightly different quantitatively. In a relatively large energy window the conductance profiles show similar patterns with some individual peaks specific to $N$, the number of $\mathrm{Si}$ atoms. It should be noted that in the given energy intervals the value of conductance does not exceed $3 G_{0}$, which is the value for the infinite $\mathrm{Si}$-LC. The conductance values at the Fermi level do not exhibit a monotonic trend but an oscillatory behavior is evident for $N>5$ as seen in Fig. 7(c). For $N \geqslant 6$, Si chains with even $N$ have higher equilibrium conductance values than those of odd-numbered chains. The oscillatory variation of conductance is evident even with the nonoptimized geometries (see the dashed curve). Similar oscillatory variation in the conductance of carbon atomic chains, depending on the number of atoms in the chain being odd or even, has also been observed in some previous studies. ${ }^{2,57,58}$ Interestingly, it is just the opposite for the case of jellium electrodes and carbon atomic wires where oddnumbered wires show a higher conductance than evennumbered ones, as calculated by Lang and Avouris. ${ }^{57,58}$ The interaction of the atoms in the chain with the $\mathrm{Al}$ electrodes turns the discrete levels of the chain into broadened resonances. The material of electrodes, their detailed atomic structure, and the geometry of contact should all affect the quantitative results of conductance and determine whether the odd-numbered or even-numbered chains have higher conductance values.

The stretching of atomic chains under tension may have drastic effects on properties of these tiny systems, including their electronic conductance. First, we have simulated the effect of elongation on the equilibrium conductance of finite linear chains using a five-atom Si-LC. It is assumed that pulling the rigid Al electrodes apart uniformly stretches all the bonds in the wire including the ones between the wire and the electrodes. The resulting variation of conduction with strain $\varepsilon$ is plotted in Fig. 7(d). Intuitively, one would expect that increasing the interatomic distances in the chain should decrease the conductance since the overlapping between the atomic orbitals decreases with increasing separation. Indeed, a monotonic decrease of conductance is observed from $2.32 G_{0}$ to $1.21 G_{0}$ as the chain is stretched from equilibrium up to $8 \%$ strain. However, for larger values of $\varepsilon$ we observe two resonance peaks at $\varepsilon=10 \%$ and $14 \%$. Such interesting variations of conductance are again due to the detailed effects of charging and broadening in the molecular energy levels of the chain when coupled to the electrodes. For carbon chains in contact with Al electrodes having the same structural forms, Larade et al. ${ }^{6}$ have shown that oscillatory variation of transmission coefficients (and hence the conductance) with the chain-electrode distance $d_{e}$ is related to how well the scattering states (in the electrodes) overlap with the highest occupied molecular orbital and the lowest 
unoccupied molecular orbital of the chain. The same mechanism is responsible for the calculated conductance variations of strained Si chains. An increase of conductance in stretched $\mathrm{Al}$ atomic wires is also reported by Okano et al. ${ }^{10}$ who attribute these conductance variations to peculiar features of the electron states of atomic wires consisting of group-III or group-IV atoms.

We have also considered the conductance of zigzag $\mathrm{Si}$ chains by fixing the Al electrode-electrode distance at a given value and by optimizing all the Si atomic positions. In panels (e) and (f) of Fig. 7 we plot the variations of the change in the total energy and the conductance of seven and eight-atom Si zigzag chains as functions of the interelectrode distance ( $L$, see Fig. 2). The total energy of the structures displays minima at such $L$ values allowing zigzag Si chains with approximately WZ forms (with $\sim 120^{\circ}$ apex angles). The variation of conductance is more interesting showing a clear odd-even $N$ disparity. The conductance of the sevenatom $\mathrm{Si}$ chain monotonically drops from $1.38 G_{0}$ to $0.86 G_{0}$ as $L$ is increased from $13.8 \AA$ to $15.5 \AA$. On the other hand, conductance of the eight-atom $\mathrm{Si}$ chain displays an opposite trend, increasing from $1.54 G_{0}$ at $L=16.0 \AA$ to $1.99 G_{0}$ at $L$ $=18.3 \AA$. It is interesting to note that the even $N$ chain $(N$ $=8$ ) has larger conductance values than those of the odd- $N$ chain $(N=7)$ for all reasonable values of $L$, consistent with the conductance values calculated in the LC geometries [panel (c)].

In nanoscales, detailed atomic configuration of the system at hand has important implications. For example, the type of the terminal atom in a compound wire or the binding site of a chain on the electrodes changes the system characteristics. The binding of external atoms on nanowires, and impurities as well modify the structural and electronic properties. In Fig. 8 we consider a few such cases with regard to the conductance of $\mathrm{Si}$ and AlP linear atomic chains. The top two panels compare the conductance of AlP chains in contact with the hollow site of $\mathrm{Al}$ electrodes with either $\mathrm{Al}$ or $\mathrm{P}$ end atoms. Since Al electrodes make better contacts with $\mathrm{Al}$ atoms than $\mathrm{P}$, the ballistic conductance of the latter chain $\left(0.27 G_{0}\right)$ drops to half the value of the former one $\left(0.53 G_{0}\right)$. The dependence of conductivity on contact geometry of the electrodes is evident in panel (c) of Fig. 8. When the surface layers of the $\mathrm{Al}$ electrodes are taken to be with five atoms (lower configuration in Fig. 2), the ends of the silicon chain bind to the central $\mathrm{Al}$ atoms and the configuration is referred to as top-site binding. We find that the equilibrium conductance of this configuration is $2.08 G_{0}$, less than the corresponding value in hollow-site binding, $2.32 G_{0}$.

We have considered the reaction of $\mathrm{Si}$ atomic chains to external adatoms of $\mathrm{O}$ and $\mathrm{C}$. When an $\mathrm{O}$ atom is brought to the central vicinity of an otherwise linear chain of $\mathrm{Si}$ atoms, structural relaxations show that the external atom is chemisorbed to one of the Si atoms of the chain by displacing the central atom and buckling the chain. ${ }^{59}$ Such chemisorption processes can be viewed as the functionalization of the atomic chain. It should be noted that the adsorbed atoms change the spherical symmetry of Si-LC and trigger a structural transition into a zigzag chain which has a higher binding energy (compare the $E_{b}$ values of $\mathrm{Si}$ atomic chains given Table I). Fuctionalization of a five-atom Si chain by the ad-

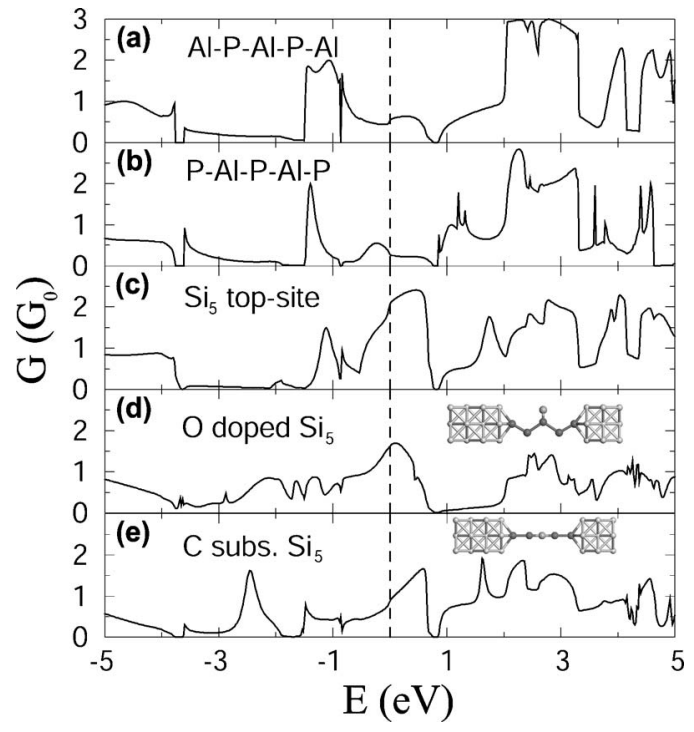

FIG. 8. Conductance plots of several configurations of atomic wires in between $\mathrm{Al}$ (100) electrodes; (a) five-atom AlP-LC with Al ends, (b) five-atom AlP-LC with P ends, (c) five-atom Si-LC bound to a top site of $\mathrm{Al}$ electrodes, (d) five-atom $\mathrm{Si}$ chain doped with $\mathrm{O}$, (e) substitutionally $\mathrm{C}$ doped five-atom $\mathrm{Si}$ chain. Fermi level is set to zero in all plots.

sorbtion of an $\mathrm{O}$ atom at the top site of Si drops the ballistic conductance value from $2.32 G_{0}$ to $1.62 G_{0}$ at the Fermi level [compare Fig. 7(b) and Fig. 8(d)].

Functionalization of the $\mathrm{Si}$ atomic chain with a carbon atom resulted in the insertion of the carbon atom into the chain through structural relaxation. The carbon atom first formed strong bonds with two neighboring $\mathrm{Si}$ atoms of the chain by breaking the $\mathrm{Si}-\mathrm{Si}$ bond. Then the carbon atom is pulled to align with the chain due to its strong tendency to linearize its double bonds with the silicon atoms. To demonstrate that incorporation of a carbon atom in a silicon chain is energetically favored, the total energy of a six-atom Si-LC segment plus an isolated carbon atom is compared to that of a $\mathrm{Si}_{3}-\mathrm{C}-\mathrm{Si}_{3}$ chain, and the latter is found to have $8.7 \mathrm{eV}$ lower energy. Therefore, for transport calculations we considered a case where a $\mathrm{C}$ atom is treated as a substitutional impurity formed during the synthesis of the $\mathrm{Si}$ chain. Figure 8(e) shows the conductance spectrum of a five-atom LC with $\mathrm{Al}$ electrodes, where the central $\mathrm{Si}$ atom is replaced by a C. It is seen that the $\mathrm{C}$ impurity decreases the conductance of the Si chain significantly $\left[G\left(E_{F}\right)=0.88 G_{0}\right]$, consistent with the fact that the infinite LC of the $\mathrm{SiC}$ compound is a good insulator with $E_{g}=2.4 \mathrm{eV}$.

\section{CONCLUSIONS}

We have presented a study of various atomic chains made of group-IV elements $\mathrm{Si}, \mathrm{Ge}$, and $\mathrm{Sn}$, and binary compounds of III-V and II-VI group elements. First-principles DFT calculations predict that stable atomic chain structures of these materials can be formed. The bonding character observed in atomic strings of group-IV elements and III-V and II-VI compounds makes these structures have substantially differ- 
ent properties from their bulk crystals. Several chain structures like $\mathrm{Si}, \mathrm{Ge}, \mathrm{Sn}, \mathrm{GeSi}, \mathrm{GaAs}, \mathrm{InSb}$, and CdTe are found to form metallic chains, whereas others like $\mathrm{SiC}, \mathrm{GaN}$, and $\mathrm{ZnSe}$ are found semiconducting or insulating with varying energy band gaps. Other than a linear chain, which corresponds to a state of local minimum in the total energy of the structure, most compound atomic wires tend to make wide zigzag chain structures. Elemental atomic chains of $\mathrm{Si}, \mathrm{Ge}$, and $\mathrm{Sn}$, as well as the GeSi compound, may also form in narrow zigzag configuration. The compound and atomic strings are found to be relatively strong against long-range longitudinal perturbations, such as AlP and CdTe chains supporting axial strains of more than $30 \%$.

Finite-sized AlP and Si linear chains exhibit interesting variations of ballistic conductance values as functions of a number of atoms in the chain and applied strain. The conductance oscillations as a function of number of atoms are observed for $\mathrm{Si}$ chains in between $\mathrm{Al}$ electrodes. For that specific type and geometry of electrodes, even-numbered Si chains are found to have higher conductance than oddnumbered chains. Under applied strain, the conductance of the five-atom $\mathrm{Si}$ chain changes nontrivially showing some resonance peaks.

With possible structural and chemical functionalizations, and through the formation of grid and network structures, $\mathrm{Si}$ atomic strings may have important potential applications that deserve further studies. Stable atomic strings predicted by first-principles, structure-optimized calculations indicate that such structures can, in fact, exist. Their interesting physical properties are hoped to motivate further experimental research for their synthesis.

\section{ACKNOWLEDGMENTS}

S.C. acknowledges partial support by TÜBA (The Turkish Academy of Sciences). Part of the computations has been carried out at ULAKBIM Computer Center.
*Corresponding author. Electronic address: ciraci@fen.bilkent.edu.tr

${ }^{1}$ H. Ohnishi, Y. Kondo, and K. Takayanagi, Nature (London) 395, 780 (1998).

${ }^{2}$ A. I. Yanson, G. R. Bollinger, H. E. van den Brom, N. Agrait, and J. M. van Ruitenbeek, Nature (London) 395, 783 (1998).

${ }^{3}$ D. R. Bowler, J. Phys.: Condens. Matter 16, R721 (2004).

${ }^{4}$ J.-L. Mozos, C. C. Wan, G. Taraschi, J. Wang, and H. Guo, Phys. Rev. B 56, R4351 (1997).

${ }^{5}$ P. Sen, S. Ciraci, A. Buldum, and I. P. Batra, Phys. Rev. B 64, 195420 (2001).

${ }^{6}$ B. Larade, J. Taylor, H. Mehrez, and H. Guo, Phys. Rev. B 64, 075420 (2001).

${ }^{7}$ N. Agrait, C. Untiedt, G. Rubio-Bollinger, and S. Vieira, Phys. Rev. Lett. 88, 216803 (2002).

${ }^{8}$ F. J. Ribeiro and M. L. Cohen, Phys. Rev. B 68, 035423 (2004).

${ }^{9}$ F. Picaud, V. Pouthier, C. Girardet, and E. Tosatti, Surf. Sci. 547, 249 (2003).

${ }^{10}$ S. Okano, K. Shiraishi, and A. Oshiyama, Phys. Rev. B 69, 045401 (2004).

${ }^{11}$ E. Z. da Silva, F. D. Novaes, A. J. R. da Silva, and A. Fazzio, Phys. Rev. B 69, 115411 (2004).

${ }^{12}$ O. Gülseren, F. Ercolessi, and E. Tosatti, Phys. Rev. Lett. 80, 3775 (1998).

${ }^{13}$ Y. Oshima, A. Onga, and K. Takayanagi, Phys. Rev. Lett. 91, 205503 (2003).

${ }^{14}$ R. T. Senger, S. Dag, and S. Ciraci, Phys. Rev. Lett. 93, 196807 (2004).

${ }^{15}$ A. Stalder and U. Dürig, Appl. Phys. Lett. 68, 637 (1996).

${ }^{16}$ H. Mehrez and S. Ciraci, Phys. Rev. B 56, 12632 (1997).

${ }^{17}$ N. Agrait, J. G. Rodrigo, and S. Vieira, Phys. Rev. B 47, R12345 (1993).

${ }^{18}$ N. Agrait, G. Rubio, and S. Vieira, Phys. Rev. Lett. 74, 3995 (1995).

${ }^{19}$ S. Ciraci and E. Tekman, Phys. Rev. B 40, R11969 (1989).

${ }^{20}$ E. Tekman and S. Ciraci, Phys. Rev. B 43, 7145 (1991).
${ }^{21}$ A. I. Yanson, G. R. Bolliger, H. E. van der Brom, N. Agraït, and J. M. van Ruitenbeek, Nature (London) 395, 783 (1998).

${ }^{22}$ S. Tongay, R. T. Senger, S. Dag, and S. Ciraci, Phys. Rev. Lett. 93, 136404 (2004).

${ }^{23}$ For a recent review, see S. Ciraci, A. Buldum, and I. P. Batra, J. Phys.: Condens. Matter 13, R537 (2001).

${ }^{24}$ S. Ciraci, S. Dag, T. Yildirim, O. Gulseren, and R. T. Senger, J. Phys.: Condens. Matter 16, R901 (2004).

${ }^{25} \mathrm{G}$. Roth and H. Fischer, Organometallics 15, 5766 (1996).

${ }^{26}$ X. Zhao, Y. Ando, Y. Liu, M. Jinno, and T. Suzuki, Phys. Rev. Lett. 90, 187401 (2003).

${ }^{27}$ R. O. Jones and G. Seifert, Phys. Rev. Lett. 79, 443 (1997).

${ }^{28}$ A. Abdurahman, A. Shukla, and M. Dolg, Phys. Rev. B 65, 115106 (2002).

${ }^{29}$ X. T. Zhang, Z. Liu, K. M. Ip, Y. P. Leung, Quan Li, and S. K. Hark, J. Appl. Phys. 95, 5752 (2004).

${ }^{30}$ S. Bhunia, T. Kawamura, Y. Watanabe, S. Fujikawa, and K. Tokushima, Appl. Phys. Lett. 83, 3371 (2003).

${ }^{31}$ M. P. Persson and H. Q. Xu, Appl. Phys. Lett. 81, 1309 (2002).

${ }^{32}$ Y. Cui and C. M. Lieber, Science 291, 851 (2001).

${ }^{33}$ B. J. Ohlsson, M. T. Bjrk, M. H. Magnusson, K. Deppert, L. Samuelson, and L. R. Wallenberg, Appl. Phys. Lett. 79, 3335 (2001).

${ }^{34}$ Y. H. Gao, Y. Bando, and T. Sato, Appl. Phys. Lett. 79, 4565 (2001).

${ }^{35}$ Yi Cui, Lincoln J. Lauhon, Mark S. Gudiksen, Jianfang Wang, and Charles M. Lieber, Appl. Phys. Lett. 78, 2214 (2001).

${ }^{36}$ Sung-Wook Chung, Jae-Young Yu, and James R. Heath, Appl. Phys. Lett. 76, 2068 (2000).

${ }^{37}$ X. Duan and C. M. Lieber, Adv. Mater. (Weinheim, Ger.) 12, 298 (2000).

${ }^{38}$ X. Duan, J. Wang, and C. M. Lieber, Appl. Phys. Lett. 76, 1116 (2000).

${ }^{39}$ P. Soukiassian, Mater. Sci. Eng., B 96, 115 (2002).

${ }^{40}$ M. Kawamura, N. Paul, V. Cherepanov, and B. Voigtländer, Phys. Rev. Lett. 91, 096102 (2003). 
${ }^{41}$ Y. Zhao and B. I. Yakobson, Phys. Rev. Lett. 91, 035501 (2003).

${ }^{42}$ B. X. Li, P. L. Cao, R. Q. Zhang, and S. T. Lee, Phys. Rev. B 65, 125305 (2002).

${ }^{43}$ D. D. D. Ma, C. S. Lee, F. C. K. Au, S. Y. Tong, and S. T. Lee, Science 299, 1874 (2003).

${ }^{44}$ Properties of finite-size atomic chains can be deduced from the calculated properties of infinite, periodic chains except for end effects. While the characteristics of possible interconnect or device applications of these chains can be modified depending on their size, doping, type of electrodes they are connected to, the substrate they are placed on, or encapsulation, the present study aims to reveal their properties when they are freestanding or suspended between two electrodes. We believe that those basic properties are essential in designing new nanodevices.

${ }^{45}$ M. C. Payne, M. P. Teter, D. C. Allen, T. A. Arias, and J. D. Joannopoulos, Rev. Mod. Phys. 64, 1045 (1992).

${ }^{46}$ Numerical calculations have been carried out by using the VASP software package. G. Kresse and J. Hafner, Phys. Rev. B 47, R558 (1993); G. Kresse and J. Furthmüller, ibid. 54, 11169 (1996).

${ }^{47}$ P. Hohenberg and W. Kohn, Phys. Rev. 136, B864 (1964); W. Kohn and L. J. Sham, ibid. 140, A1133 (1965).

${ }^{48}$ J. P. Perdew, K. Burke, and M. Ernzerhof, Phys. Rev. Lett. 77, 3865 (1996).

${ }^{49}$ J. P. Perdew and A. Zunger, Phys. Rev. B 23, 5048 (1981).

${ }^{50}$ For recent progress in the $G W$ method, see, for example, F. Aryasetiawan and O. Gunnarsson, Rep. Prog. Phys. 61, 237 (1998). The $G W$ method has recently been applied in a study of $(n, 0)$ zigzag carbon nanotubes [T. Miyake and S. Saito, Phys. Rev. B 68, 155424 (2003)], and the calculated corrections are found to be small. On the other hand, important excitonic effects in carbon nanotubes have been explained by performing firstprinciples many-body Green's function calculations [C. D. Spataru, S. Ismail-Beigi, L. X. Benedict, and S. G. Louie, Phys. Rev. Lett. 92, 077402 (2004)].

${ }^{51}$ H. J. Monkhorst and J. D. Pack, Phys. Rev. B 13, 5188 (1976).

${ }^{52}$ D. Vanderbilt, Phys. Rev. B 41, R7892 (1990).

${ }^{53}$ The methodology of the TRANSIESTA-C software package is described in M. Brandbyge, J.-L. Mozos, P. Ordejón, J. Taylor, and K. Stokbro, Phys. Rev. B 65, 165401 (2002). The software was provided by Atomistix Corp.

${ }^{54}$ In our earlier preliminary study of string structures [S. Tongay, E. Durgun and S. Ciraci, Appl. Phys. Lett. 85, 6179 (2004)], the LC structure was identified as a stable structure. Although this conclusion is essentially true for $\mathrm{C}$ and $\mathrm{BN}$, a detailed analysis of the Born-Oppenheimer surface in the current study has revealed that planar $\mathrm{WZ}$ or $\mathrm{NZ}$ structures can form in relatively lower energies for $\mathrm{Si}, \mathrm{Ge}, \mathrm{Sn}, \mathrm{GaAs}$, etc. The potential barrier between LC and WZ structures may be very small, even zero. Transverse displacements of alternating atoms can transform a freestanding LC to a WZ structure. The stability of NZ structure of compounds can be conspicuous owing to the polarity of the atoms, and nonplanar structures of the chains with lower energies can be achieved through out-of-plane displacements of the atoms in the NZ geometry.

${ }^{55}$ D. Sanchez-Portal, E. Artacho, J. M. Soler, A. Rubio, and P. Ordejon, Phys. Rev. B 59, 12678 (1999).

${ }^{56}$ P. J. Feibelman, Phys. Rev. Lett. 54, 2627 (1985).

${ }^{57}$ N. D. Lang and Ph. Avouris, Phys. Rev. Lett. 81, 3515 (1998).

${ }^{58}$ N. D. Lang and Ph. Avouris, Phys. Rev. Lett. 84, 358 (2000).

${ }^{59}$ Depending on the initial conditions, the $\mathrm{O}$ atom can alternatively be bound at a bridge site of the chain. 